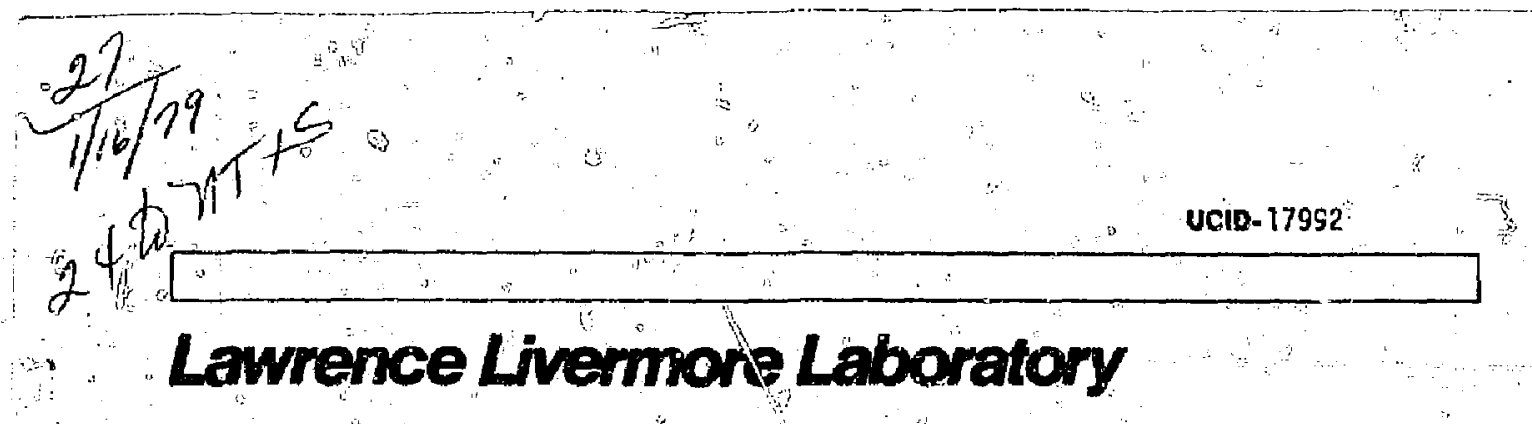

RADIAL OIFFUSION OF RESONANT PARTICLES IN TANDEN MIRRORS

Yung-Chang Lee

December 4,1978
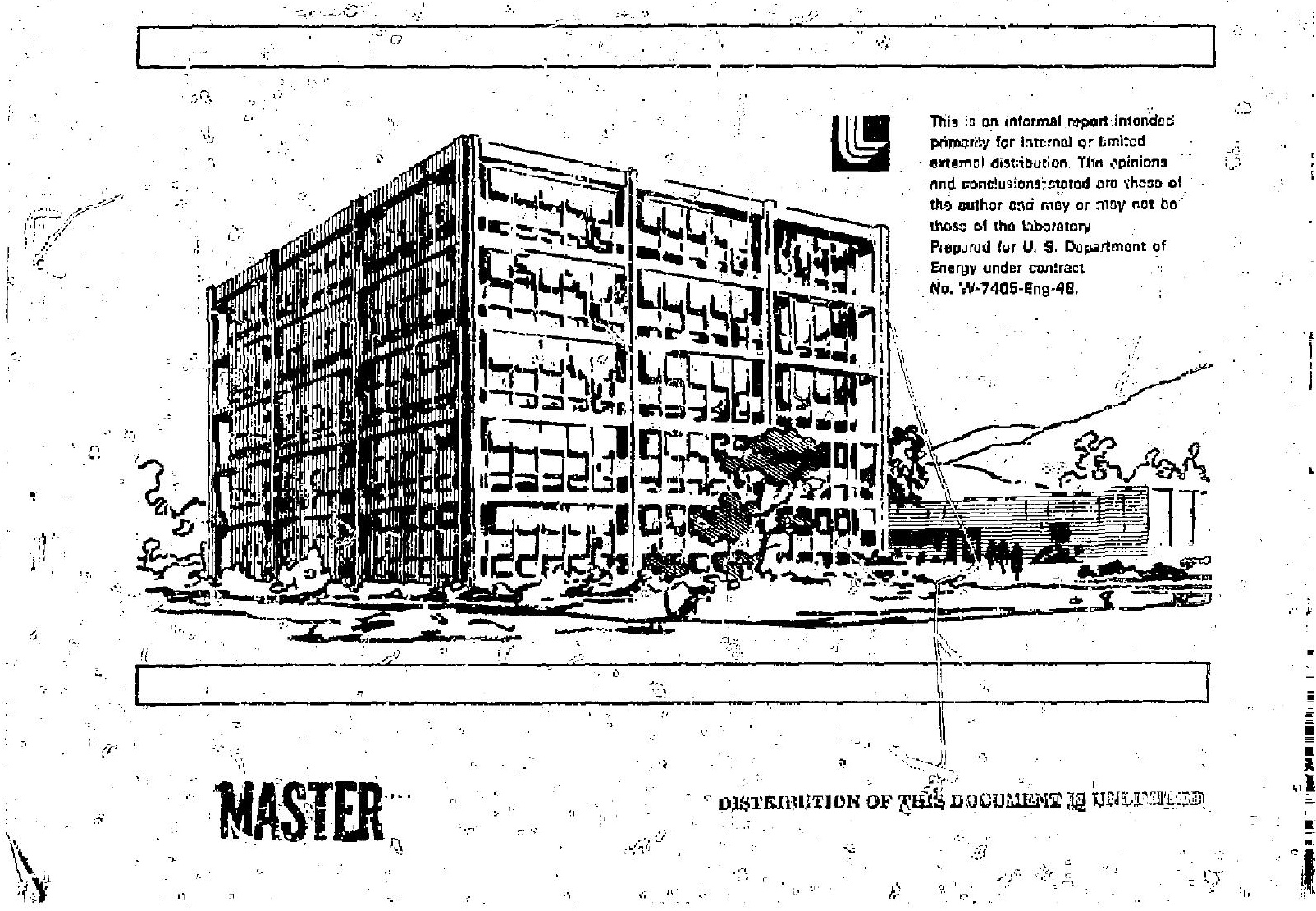


\title{
Radial Diffusion of Resonant Particles in Tandem Mirrors*
}

\author{
by \\ Yung-Chang Lee** \\ Lawrence Livermore Laboratory \\ Livermore, California 94550
}

ABSTRACT

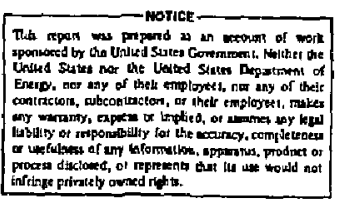

The radial diffusion of resonant particles in the banana regime and the plateau regime is derived in detail from kinetic theory. The plateau diffusion, catculated in the $\tau$-approximation, confirms the result given by Ryutov-Stupakov without derivation. The banana diffusion also agrees with the $\mathrm{R}-\mathrm{S}$ result provided the iatter is corrected for some dependence of the collision frequency on $E$ and $\phi$.

*Work performed under the auspices of the U.S. Department of Energy by the Lawrence Livemore Labsrztory under contract number W-7405-ENG-48.

* On leave from Physics Department, State University of New York at Buffalo, Amherst, New York 14260 


\section{Introduction and Summary}

Owing to the lack of axial symmetry associated with the quadruple fields in a tandem mirror system, ions can diffuse radially with an enhanced rate. ${ }^{1-3}$ Depending on whether the longitudinal bounce frequency $\omega_{\text {bounce }}$ is greater or smaller than the azimuthal drift frequency wdrift, this diffusion can be classified as the "neoclassical" diffusion and the "resonant" diffusion. In the case of $\omega_{\text {bounce }}>w{ }^{\text {drifts }}$, the radial displacements of the ions $\Delta r$ incurred in the short transition regions at either end of the tandem system tend to cancel each other because of the relative orientations of the fields in the two regions (omnigeneity), rendering the neoclassical diffusion less important. On the other hand, when $\omega_{\text {drift }}>\omega_{\text {bounce }}$ the radial displacenents at both ends can reinforce each other when the change of the azimuthal drift angie $\Delta \psi$ during the ion motion from one end to the other is just right, ie. $\Delta \psi(r, \mu, \varepsilon)=k \pi / 2, k=$ odd integers. Those particles which satisfy the above condition almost exactiy will accrue the radial excursions continuously to a large maximum value $x_{\text {res }} \sim \sqrt{r^{\circ} R \text {, }}$ where $R$ is the radius of the plasma, provided the radial drift notion is not incerrupted by collisions. Consequently, when the collision frequency $v_{i}$ is sufficiently small, ie. $v_{i} t_{\|} \ll(\Delta r / R)^{3 / 2}$, the radial diffusion is dominated ty such resonant particles with large step sizes, leading to a rate proportional to $v_{i}$ the banana diffusion. The "mildly resonant" particles which lie a little farther away from the resonance curve can also have the radial displacement built up to an appreciable value $x_{M, R}$, $\Delta r(\varepsilon / \Delta \varepsilon) \sim \Delta r\left(v_{i} t_{j}\right)^{1 / 3}$ where $\Delta \varepsilon$ is the deviation from the resonance energy $\epsilon_{k}$. When collisions are sufficiently frequent to force the resonant particles into a local Maxwellian distribution but not frequent enough to do so for the mildly resonant porticles because the latter occupy 
a larger volume in phase space, the diffusion will be dominated in the regime $(\Delta r / R)^{3 / 2} \ll v_{i} t_{\|} \ll 1$ by the mildly resonant particles - the plateau diffusion. This regime is characterized by a diffusion rate independent of $v_{i}$, and, according to present thinking, ${ }^{4}$ is most relevant to the tandern mirror system.

The kinetic-theoretic derivation of the radial diffusion can bs carried out for the different regimes separately. In the resonant plateau regime, we can pattern after baleev and Sagdeev ${ }^{5}$ in a first calculation by neglecting the $\dot{r} \frac{\partial \delta t}{\partial r}$ term in comparison with the $\dot{\psi} \frac{\partial \delta f}{\partial \psi}$ term and using the $\tau$-approximation to the collision integral in the drift-kinetic equation. The detailed expression for the diffusion coefficient contains the qualitative features expected on physical ground, $D_{p l a t} \sim(\Delta r)^{2} / t_{\|}$, and confims that given without derivation in Ref. 3. The calculation for the banana diffusion is more complicated mathematically. The $\tau$-approximation can no longer be used. Instead, recognizing the rapid variation of the resonant particle distribution across the narrow strip of resonance in phase space, we can approximate the collision integrat by just keeping the highest order derivatives in the direction perpendicular to the resonance curve. A change of varjables with one of them arranged to vary in just that direction greatly simplifies the calculation. Furthermore, we shall not neglect the $\dot{r} \frac{\partial \delta f}{\partial r}$ term in the kinetic equation since the other term, $\dot{\psi} \frac{\partial \delta f}{\partial \psi}$, proportion to $\dot{\psi}$, can vanish for particles at the turning points of the ir banana orbits. The left hand side of the kinetic equation for $\delta f \equiv f-f_{M}$, now containing derivatives of $\delta f$ with respect to both $r$ and $\psi$, can yet be simplified and reduced to one derivative (with respect to $\psi$ ) term by changing the variable $r$ to $J(r, \psi)$, a constant of the unperturbed orbital motion. However, such a change also splits the single collision term (the 
right hand side) into many terms with different derivatives. Fortunately, in the limit of rare collisiosis, a perturbation expansion in terms of $v_{1}$ can be used. The lowest order component $g^{|0\rangle}$ of $\delta f$, which is odd with respect to the sign of $\psi$, can be solved for with the help of the boundary conditions that it vanishes at the turning point where $\dot{\psi}=0$, remains finite as $\mathrm{J}+\infty$ and is continuous across the separatrix. The averaged radial flux which can be shown to depend only on $h^{(1)}$, the lowest order component of $\delta f$ that is even with respect to the sign of $\dot{\psi}$ and odd with respect to $\psi \rightarrow-\psi$, can be imediately calculated by using the known relation coupling $h^{(1)}$. to $g^{(0)}$. The resulting expression for the diffusion coefficient contains the expected qualitative features: ${ }^{6}$

$$
D_{b a n} \sim \frac{|\Delta r|^{\frac{1}{2}}\left|\frac{\partial \Delta \psi}{\partial \varepsilon}\right|}{\left|\frac{\partial \Delta \psi}{\partial r}\right|^{3 / 2} \varepsilon_{k} \nu_{j}}
$$

Although the above expression appears quite different from that given in Ref. 3 , the actual difference lies in just a modification of the dependence of $v_{j}$ on the resonant particle energy $\varepsilon_{k}$ and the electric potential $\phi$. In the high collision frequency regime, $v_{i} t_{\|} \gg 1$, the particles have to random-walk from one transition region to the other, leading to a diffusion coefficient $0_{\text {coll. }} \sim\langle\Delta r\rangle^{2} / \tau_{\text {ran.walk }} \sim\langle\Delta r\rangle^{2} / t_{\|}^{2} v_{i}$.

Section II describes the drift orbits. Section III is devoted to the kinetic-theoretic derivation of the plateau diffusion, Sec. IV to that of the banana diffusion. Some comments and remarks are made in the final section. 
II. The Drift Orbits

In the long axially symmetric central section of the tandem mirror system (see Fig. 1) the particle will drift, on account of the radial electric field and the radial gradient of the magnetic field amplitude, around the mirror axis, resulting in a change $\Delta \psi(r, \varepsilon, H)$ in the az imuthal angle as it goes from one end to the other. Owing to the axial symetry of this central section no radial drift occurs. Upon entering and re-emerging from the short transition region at either end, the particle will incur a radial displacement $\Delta r$ while the simultaneous azimuthal displacenent is negligible compared to $\Delta \psi$. As shown in Refs. 3 and $4, \Delta r$ generally depends on $r, \psi, \mu, \varepsilon$ and, in the lowest order expansion in our asymmetry parameter $R / L_{t r}$, can be written as

$$
\Delta r \approx a_{1}(r, \mu, E) \cos 2 \psi
$$

where

$$
\begin{aligned}
& a_{1} \sim t_{t r} . v_{r} \sim \frac{L_{t r}}{v_{l l}} \frac{B_{\|} \frac{B_{t}}{L_{t r}}}{B^{2}} \frac{v_{L}^{2}}{\Omega}
\end{aligned}
$$

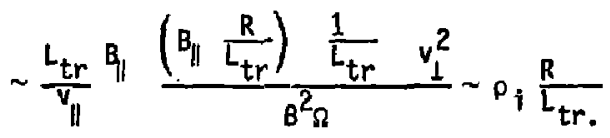

Note that $\Delta r \neq 0$ can be entirely ascribed to the asymetric quadruple field in the transition region.

In one axial round trip, the changes in $r$ and $\psi$ are given by

$$
\begin{aligned}
& r_{n+1}-r_{n}=a_{1}\left(r_{n}\right) \cos 2\left(\psi_{n}+\frac{1}{2} \Delta \psi\left(r_{n}\right)\right)-a_{1} \cos 2\left(\psi_{n}+\frac{3}{2} \Delta \psi\right)+\sigma\left(a_{1}^{2}\right) \\
& \psi_{n+1}-\psi_{n}=2 \Delta \psi\left(r_{n}\right),
\end{aligned}
$$


where the opposite signs in the two terms on the right hand side of Eq. (3) reflect the relative orfentations of the quadruple fields in the two transition regions. When $\Delta \psi$ is small, the radial displacements nearly cancel. On the other hand, when the resonance condition

$$
\Delta \psi(r, \varepsilon, \mu)=k \pi / 2, \quad k=1,2,5, \ldots .
$$

or

$$
\varepsilon=\varepsilon_{k}(r, \mu)
$$

is satisfied, they reinforce each other. When $\mu$ is given, a particle in the neighborhood of $r$ and $\varepsilon_{k}$ will have

$$
\Delta \psi\left(r+x, \varepsilon_{k}+\Delta \varepsilon, \mu\right)=k \frac{\pi}{2}+\psi_{\varepsilon}^{-} \Delta \varepsilon+\psi_{r}^{-} x+\ldots
$$

where

$$
\psi_{\varepsilon}^{-} \equiv\left[\frac{\partial \Delta \psi(r, \varepsilon, \mu)}{\partial \varepsilon}\right]_{\varepsilon=\varepsilon_{k}}, \psi_{r}^{r} \equiv\left[\frac{\partial \Delta \psi\langle r, \varepsilon, \mu\rangle}{\partial r}\right]_{\varepsilon=\varepsilon_{k}}
$$

It then follows that

$$
\begin{aligned}
& \psi_{n+2}=\psi_{n}=4 \psi_{E}^{*} \Delta \varepsilon+4 \psi_{r}^{*} x+2 k \pi, \\
& x_{n+2}-x_{n}=4(-1)^{\frac{k+1}{2}} a_{1} \sin 2 \psi,
\end{aligned}
$$

leading to the velocities

$$
\begin{aligned}
& :-\frac{\psi_{u}^{x}+\psi_{\varepsilon}^{\Delta \varepsilon}}{t_{\|}}, \\
& \dot{x}=\frac{(-1)^{\frac{k+1}{2}}}{t_{\|}} a_{1} \sin 2 \psi,
\end{aligned}
$$


where $t_{11}$ is one axial transit time. The trajectory is represented by the differential equation

$$
\frac{d \psi}{d x}=\frac{\psi_{r}^{-x}+\psi_{E}^{\Delta \varepsilon}}{(-1)^{\frac{k+1}{2}} a_{1} \sin 2 \psi},
$$

or its solution

$$
J \equiv\left(x+x_{0} \xi\right)^{2}+y(\psi)=\text { const. }
$$

where

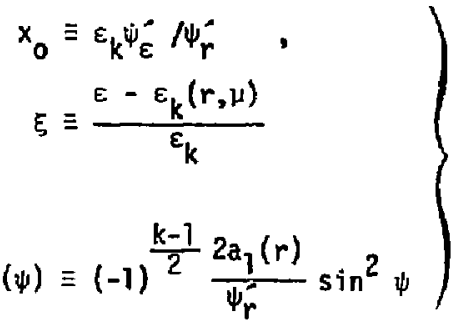

As usual, the orbits described by Eq. (13) can be divided into the banana orbits and the transiting orbits for $\mathrm{J}<\mathrm{J}_{0}$ and $\mathrm{J}>\mathrm{J}_{0}$, respectively, where $J_{0}$ is given by $\mathrm{Eq} .(16)$ and $y(\psi)$ assumed positive.

We now ask: For a particle in the neighborhood of $r$ having energy $\varepsilon_{k}(r, \mu)$ and magnetic monent $\mu$, what is tine range $\Delta x \equiv|x|_{\max }-|x|_{\text {min }}$ of its radial displacements? It is easy to see from Et. (13) that

where

$$
\begin{aligned}
& x_{\max }^{2}=J \\
& x_{\min }^{2}= \begin{cases}J=J_{0} & \text { for } J>J_{0} \\
0 & \text { for } \mathrm{J}<J_{0},\end{cases}
\end{aligned}
$$

$$
j_{0} \equiv 2\left|\frac{a_{q}}{\psi_{r}^{\prime}}\right|=x_{\text {res }}^{2}
$$


It follows that $\Delta r$ takes on its maximum value $\Delta x_{\max }=x_{\text {res }}$ when $\mathrm{J}=$ $J_{0}$. Roughly speaking we shall refer to those particles having energy $\varepsilon_{k}(r, \mu)$ and in the $x_{\text {res }}$-neighborhcod of $r$ as the "resonant" particles. We may also characterize this resonance region in terms of an energy range at given $r$,, ie. $\Delta \varepsilon<\epsilon_{\text {res }} \equiv \psi_{r}^{-} x_{\text {res }} / \psi_{\varepsilon}^{-}$, as depicted in Fig. 2. A particle in the neighborhood of $r$ but with an energy $\varepsilon$ such that $\varepsilon>\Delta \varepsilon>\Delta \varepsilon$ res will be called a "mildly resonant" (MR) particle. Its orbit can be obtained from Eqs. (10)-(13) by neglecting $\psi_{\mu}^{*} x$ compared to $\psi_{E}^{\Delta \varepsilon}$, resulting in

$$
\dot{\psi}=\psi_{\varepsilon}^{-\Delta \varepsilon / t_{\|}}
$$

and

$$
x=\frac{(-1)^{\frac{k-1}{2}} a_{1} \cos 2 \psi}{2 \psi_{\varepsilon}^{\Delta \varepsilon}}+\text { const }
$$

The corresponding radial range $\Delta x_{M R}=\left|a_{1} / 2 \psi_{\varepsilon}^{-} \Delta \varepsilon\right|$ is smaller than that for the resonant particles, ${ }^{7} x_{\text {res }}$, implying a smaller step size for diffusion. However, the energy range for the. MR-particles is much larger. The nonresonant (NR) particles with $\Delta \varepsilon \sim \varepsilon$ have $\Delta x_{\text {NR }} \sim a_{1}$, which is smaller tran $\Delta x_{M R}$. 
III Kinetic Theory-Plateau Diffusion

We first consider the case in wich collisions are sufficiently frequent that the "resonant" particles are forced into a locat Maxwellian distribution within the narrow region in phase space depizted in Fig. 2 rather than follow their unperturbed orbits to any significant degree of completion. On the other hand we assume that collisions are neg?igible for tine MR- end the NR-particles. Thus require $v_{\text {res }}^{\text {eff }} \tau_{\text {res }} \gg 1$ but $v_{i} t_{\|} \ll 1$ or $\left(a_{1} / R\right)^{3 / 2}$ $\ll v_{i} t_{\|} \ll 1$, where $\nu_{\text {res }}^{\text {eff }} \sim v_{j}\left(\varepsilon / \Delta \varepsilon_{\text {res }}\right)^{2}$ is the effective collision frequency and $\tau_{\text {res }} \sim x_{\text {res }}\left(a_{1} / t_{11}\right)^{-1}$ the drift periad for the resonant particles. With the resonance orbits sporting the largest step size demolished, the diffusion in the regime is expected to be dominated by the MR-particies. The drift-kinetic equation 8 is

$$
\dot{r} \frac{\partial f}{\partial r}+\dot{\psi} \frac{\partial f}{\partial \psi}=\left(\frac{\partial f}{\partial t}\right)_{\text {coll }} \text {. }
$$

We write the distribution function in the form

$$
f(r, \psi, \mu, \varepsilon)=f_{M}(r, \varepsilon)+\delta f(r, \psi, \mu, \varepsilon)
$$

where

$$
f_{M}=n(r)\left[\frac{m_{i}}{\tau_{\pi} \frac{T_{j}}{(r)}}\right]^{3 / 2} \exp \left[-\frac{\varepsilon-e \phi(r)}{T_{i}(r)}\right]
$$

is the local Maxwellian distribution including, in particular, the thermalized resonant particles and of describes essentially the Mr-particles. Using Eqs. (17) and (11) for MR-particles in the plateau regimes we can estimate the ratio $\dot{r} / r \dot{\psi}$ and show that $\left(a_{1} / R\right)^{1 / 2}>\dot{r} / r \dot{\psi}>a_{1} / R$, where we have also used $\left[\nu_{i}(\varepsilon / \Delta \varepsilon)^{2}\right] \cdot\left[\Delta x_{M R}\left(a_{1} / t_{\|}\right)^{-1}\right] \sim 1$ or $\Delta \varepsilon / \varepsilon \approx\left(v_{i} t_{\|}\right)^{1 / 3}$ for the group of MR-particles contributing dominantly to the diffusion. Substitution of Eqs. (17), (11) into the linearized $\mathrm{Eq} .(18)$ and the neglect of $\dot{r} \frac{\partial \delta f}{\partial r}$ compared to $r \dot{\psi} \frac{\partial \delta f}{r \partial \psi}$ lead to the simpinfied equation 
10

$$
\frac{1}{t_{\|}} \psi_{\varepsilon}^{-}\left(\varepsilon-\varepsilon_{k}\right) \frac{\partial \delta f}{\partial \psi}+\frac{a_{1}}{t_{\|}}(-1)^{\frac{k+1}{2}} \sin 2 \psi \frac{\partial f_{y}}{\partial r}=-v \delta f
$$

where the collision integral has been written in the $\tau$-approximation. 5 The reason why can use s'ich a crude approximation is that of for the MRparticles is expected to vary less sharply over a larger volume of phase space (than the resonant particles, say), rendering the exact form of the collision term less crucial. ${ }^{9}$ Equation (20) can be solved immediately to yield

$$
\left.\delta f=\frac{(-1)^{\frac{k+1}{2}} a_{1}}{2} \mid \frac{e^{2 i \psi}}{2 \psi_{\varepsilon}^{-}\left(\varepsilon^{-} \varepsilon_{k}\right)-i_{\nu} t_{\|}}+\frac{e^{-2 i \psi}}{2 \psi_{\varepsilon}^{-}\left(\varepsilon-\varepsilon_{k}\right)+i v t_{l}}\right\} \frac{\partial f_{H}}{\partial r}
$$

The radial particle flux averaged over $\psi$ can be written as

$$
\begin{aligned}
\left\langle a_{r}\right\rangle & =\int\langle\dot{r} d n\rangle \\
& =\sum_{k=1,3,5, \ldots} \int_{d \mu} d m_{i} \frac{2 \pi H}{L_{i}^{2}}(-1)^{\frac{k+1}{2}} a_{T}\langle f \sin 2 \psi\rangle,
\end{aligned}
$$

where the different resonances are assumed non-overlapping and contribute independently to the flux. Since $\left\langle f_{M} \sin 2 \psi\right\rangle=0$, the integrand in Eq. (22) is proportional to $\langle\delta f \sin 2 \psi\rangle$, given by

$$
\langle\delta f \sin 2 \psi\rangle=-\frac{1}{4 i}(-1)^{\frac{k+1}{2}} a_{1} \frac{\partial f_{M}}{\partial r}\left[\frac{1}{2 \psi_{\varepsilon}\left(\varepsilon-\varepsilon_{k}\right)-i v t_{i}}-c . c .\right] \text {. }
$$


We may now substitute the above into $\mathrm{Eq}$. (22) and take the limlt

$v t_{11} \rightarrow 0+$ to obtain

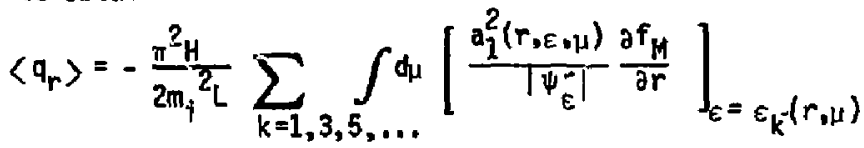

Note that the limit of vanishing collision frequency can only be taken at this stage after making all the provisions which ensured that Eq. (20) and its solution sf are for the MR-particles and not for the resonant particles. Dtherwise, the $0^{+}$-collision frequency would certainly lead to diffusion dominated by the resonant particles with large step sizes in their rarely perturbed orbits rather than by the IR-particles in theirs. The fact that $\left\langle q_{r}\right\rangle$ is independent of the collision frequency comes about, ${ }^{9}$ rot because collisions play no role, but because thie width $\Delta \varepsilon$ of the distribution scales as $v^{1 / 3}$ while its magnitude scales as $v^{-1 / 3}$, as in the case of Landau damping. 10

Qualitatively the diffusion coeffictent is governed by those MR-particles which ho $\approx$ orift times $\tau_{M R} \approx \Delta x_{M R}\left(a_{1} / t_{j}\right)^{-1}$ comparable to the invorse effective collision frequency $\left(v_{M R}^{e f f}\right)^{-1} \approx\left(v_{i} \frac{\varepsilon^{2}}{\Delta \varepsilon^{2}}\right)^{-1}$

Thus,

$$
D_{P 1 a t} \sim\left(\Delta x_{M R}\right)^{2}(\Delta \varepsilon / \varepsilon) \underset{V_{M R}}{\text { eff }} \sim a_{1}^{2} / t_{\mid l} \text {. }
$$

which is consistent with Eq. (23). Our result also confirms the one given without derivation in Ref. 3 . 


\section{Kinetic Theory - Banana Biffusion}

In the regime $v_{\text {res }}^{\text {eff }} \tau_{\text {res }} \ll 1$ or $\left(a_{1} / R\right)^{3 / 2} \gg v_{1} t_{11}$ the resonant particles only suffer rare collisians and are expected to dominate the diffusion because of their large step sizes in the unperturbed orbits. As discussed in Sec. II, the width of the resonant particle distribution is $\Delta \varepsilon_{\text {res }}=\frac{\psi_{\varepsilon} x_{\text {res }}}{\psi_{\varepsilon}^{r}}$ wich is extremely small. Therefore, the deviation of from the loca? Maxwellian distribution will vary sharply across this narrowresonance strip. This fact may be exploited to approximate the collision integral. At a given point $r$, the resonance strip runs along the curve $E$ $=\varepsilon_{k}(r, \mu)$ in the $(\mu, \varepsilon)-p l a n e$. If we perform a change of variables from $(\mu, \varepsilon)$ to $\left(\mu, \xi=\frac{\varepsilon-\varepsilon_{k}(r, \mu)}{\varepsilon_{k}}\right)$ the resonance region will be a straight strip along the $\xi=0$ axis, essentially independent of $\mu$ in the $(\mu, \xi)$ plane. In the collision integral involving derivatives with respect to the velocity components we may first transform them into derivatives with respect to $\mu$ and $\xi$ and then use the fact that $\frac{\partial}{\partial \mu} \ll \frac{\partial}{\partial \xi}$ to eliminate all but the second derivative terms with respect to $\xi$.

The linearized drift-kinetic equation appropriate for the resonant particles takes the form

$$
\frac{\omega}{r}\left[\frac{\partial J}{\partial \psi} \frac{\partial f_{M}}{\partial r}+\frac{\partial J}{\partial \psi} \frac{\partial \delta f}{\partial x}-\frac{\partial J}{\partial x} \frac{\partial \delta f}{\partial \psi}\right]=v_{i}(r, \mu) \frac{\partial^{2} \delta f\left(x_{2}\left(k_{2}, E\right)\right.}{\partial \xi^{2}}
$$

where the collision term on the right hand side is derived in the Appendix. In Eq. (24) the velocities $\dot{r}$ and $\dot{\psi}$ given by Eqs. (10) and (11) are now expressed in terms of the invariant $J(x ; \psi, \xi)$ of Eq. (13). The variable $\mu$ actualiy plays the role of a parameter and will often not be written out explicitly for convenience. Also, $r$ refers to a fixed spatial point while $x$ is the variable; $: \omega \equiv=m_{r} / 2 t_{\|}$. 
We construct from of two functions in terms of the variables $(u, \psi, \xi)$ where $u=x+x_{0} \xi \propto \dot{\psi}_{3}$

$$
\begin{aligned}
& g(u, \psi, \xi)=\frac{1}{2}[\delta f(u, \psi, \xi)-\delta f(-u, \psi, \xi)], \\
& h(u, \psi, \xi)=\frac{1}{2}[\delta f(u, \psi, \xi)+\delta f(-u, \psi, \xi)],
\end{aligned}
$$

which nave definite symmetry with respect to the change of sign of $\dot{\psi}$. At the turning point of a banana orbit where $u=\dot{\psi}=0$ or $\psi=\psi_{\text {turn }}$ with $J-y\left(\psi_{\text {turn }}\right)=0$, we see that

$$
g(u=0, \psi, \xi)=0 \text {. }
$$

The left hand side of Eq. (24) contains derivative terms with respect to both $x$ and $\psi$. We may reduce it to one derivative term by making a further change ${ }^{11}$ of variables from $(u, \psi, \xi)$ to $\left(J=u^{2}+y(\psi), \psi, \xi\right)$, exploiting the property of $\mathfrak{J}$ as an invariant of the unperturbed motion. Equation (24) hen gives rise to two coupled equations 12 for $g$ and $h$ :

$$
\begin{aligned}
-\frac{\omega}{r} \frac{\partial J}{\partial x} \frac{\partial g\left(J_{2}, \xi \xi\right)}{\partial \psi} & =-\frac{\omega}{r} \frac{\partial J}{\partial \psi} \frac{\partial f_{M}}{\partial r}+ \\
& +v_{i}\left\{\left[x_{0}^{2}\left(2 u \frac{\partial}{\partial J}\right)^{2}+\frac{\partial^{2}}{\partial \xi^{2}}\right] h+4 x_{0} \frac{\partial}{\partial \xi}\left[u \frac{\partial}{\partial J} g\right]\right\},
\end{aligned}
$$

$-\frac{\omega}{r} \frac{\partial J}{\partial x} \frac{\partial h\left(J, \psi_{2} \xi\right)}{\partial \psi}=v_{i}\left\{\left[x_{0}^{2}\left(2 u \frac{\partial}{\partial \cdot \partial} j^{2}+\frac{\partial^{2}}{\partial \xi^{2}}\right] g+4 x_{0} \frac{\partial}{\partial \xi}\left[u \frac{\partial h}{\partial J}\right]\right\}\right.$,

where

$$
\begin{aligned}
& u=\sigma \sqrt{J-y(\omega)} \\
& \sigma=\operatorname{sign}\left(x+x_{0} \xi\right) .
\end{aligned}
$$

Although the left hand sides of Eqs. (27) and (28) are simplified by the transformation the collision terms on the right hand sides have proliferated. These equations still involve three variables $\psi, J, \xi$ in an essential way. 13 The real simplification achieved is in shifting the complication from the left hand side to the teras multiplified by the small parameter $v_{i}$ 
on the right hand side. We solve for $g$ and $h$ in the form of perturbation expansions:

$$
s=g_{0}+v^{2} g_{2}+\ldots, h=w_{1}+\ldots .
$$

In the $v^{\circ}$ order, the equation

$$
\frac{\partial g_{0}(J, \psi, \xi)}{\partial \psi}=-\frac{\sigma \frac{\partial y}{\partial \psi} \frac{\partial f_{M}}{\partial r}}{2 \sqrt{J}-\bar{y}}
$$

can be iminediately integrated to yield

$$
g_{0}=-\sigma \sqrt{S-y(\psi)} \frac{\partial f_{M}}{\partial r}+c(J, \xi)
$$

where $C(J, \xi)$ is to be determired from boundary conditions. This function $C(J, \xi)$ takes on different forms for banana (trapped) particles and transiting particles. The separatrix is determined by

$$
J=J_{c} \equiv \max y(\psi)= \begin{cases}2\left|\frac{a_{1}}{\psi_{r}^{2}}\right|, & \text { when }(-1)^{\frac{k-1}{2}} a_{1} / \psi_{r}>c \\ 0, & \text { when }(-1)^{\frac{k-1}{2}} a_{1} / \psi_{r}<0\end{cases}
$$

For the banana particles (indexed by $b$ ) or when $J<J_{c}$, the boundary condition (26) at the turning point $\psi=\psi_{\text {turn }}$ applies, leading to $C^{b}(J, \xi)=0$ or

$$
g_{0}^{(b)}=-\sigma \sqrt{J-y(\psi)} \frac{\partial f_{M}}{\partial r}
$$

For the transiting particles (indexed by $t$ ) or when $J>J_{c}$ we have to resort to $E q .(28)$ for the determination of $c^{(t)}\left(J, \xi_{3}\right)$. Substitution of Eq. (30) into Eq. (28) yields, to order $v^{1}$,

$$
\frac{\partial h_{1}^{(t)}(J, \psi, \xi)}{\partial \psi}=\frac{-r \nu_{i} \sigma}{2 \omega} \frac{1}{\sqrt{J-y}}\left[4 x_{0}^{2}\left(u \frac{\partial}{\partial j}\right)^{2}+\frac{\partial^{2}}{\partial \xi^{2}}\right] g_{0}^{(t)}(J, \psi, \xi) .
$$


Upon integrating over $\psi$ from $-\pi / 2$ to $\pi / 2$ the pertadicity of the transiting particles leads to, as long as $v_{i}$ does not vanish identically,

$$
4 x_{0}^{2} \frac{\partial}{\partial J}\left[\int_{-\pi / 2}^{\pi / 2} d \psi \sqrt{J-y(\psi)} \frac{\partial c^{(t)}}{\partial J}\right]+\frac{\partial^{2} c^{(t)}}{\partial \xi^{2}} \int_{-\pi / 2}^{\pi / 2} \frac{d \psi}{\sqrt{J-y(\psi)}}=0,
$$

Which can be solved by separation of variables. However, the joining ${ }^{14}$ of: $g_{0}^{(b)}$ to $g_{0}^{(t)}$ at $J=l_{c}$ requires that $c(t)$ be independent of $\xi$ since $g_{0}(b)$ is independent of $\xi$. Thus $w^{3}$ obtain

$$
c^{(t)}(J)=k \int_{J_{c}}^{J} \frac{d J}{E(J)}
$$

where

$$
E(J) \equiv \int_{\pi / 2}^{\pi / 2} d \psi \sqrt{J-y(\psi)}
$$

To determine the constant $K$ in Eq. (35) we require that $g_{0}(t)(J, \psi, \xi)$ be finite as $\mathrm{J} \rightarrow \infty$, yielding $\mathrm{K}=\sigma \frac{\pi}{2} \frac{\partial f_{\mathrm{M}}}{\partial \mathrm{r}}$. Hence, 15

$$
g_{0}^{(t)}=-\sigma\left[\sqrt{J-y(\psi)}-\frac{\pi}{2} \int_{d_{C}}^{J} \frac{d J}{E(J)}\right] \frac{\partial f_{M}}{\partial r} \text {. }
$$

In the limit $\mathbf{J} \rightarrow \infty$,

$$
\lim _{j \rightarrow \infty} g_{0}^{(t)}(J, \psi)=\sigma \frac{\pi}{2} \frac{\partial f_{M}}{\partial r}\left[\int_{J_{c}}^{\infty} d v\left(\frac{1}{E(J)}-\frac{1}{\pi J^{1 / 2}}\right)-\frac{2}{\pi} J_{c}^{1 / 2}\right],
$$

which is finite and independent of $\psi, \xi$.

We now turn to the calculation of the radial flux. Equation (22) gives

$$
\left\langle q_{r}\right\rangle=-\frac{\pi H}{m_{i}^{2} L} \sum_{k=1,3,5, \ldots}\left\langle\int \psi_{r}^{-} \frac{\partial J}{\partial \psi} \delta f d \mu d \varepsilon\right\rangle
$$


With $\delta f=g+h$ according to Eq. $(25)$ and the fact that $g_{0}$ is even while $\frac{\partial J}{\partial \psi}$ is odd in $\psi$ the $\psi$-averaged flux can be written as

$$
\left\langle q_{r}\right\rangle=-\frac{\pi H}{m_{i}^{2} L} \sum_{k} \frac{1}{2 \pi} \int_{-\pi}^{\pi} d \psi \int d \mu \int d \xi \varepsilon_{k} \psi_{r}^{-} \frac{\partial y}{\partial \psi} h(J, \psi, \xi)
$$

where we have used the variable $\xi$ rather than $\varepsilon$ in the integration. We shall argue that $h(J, \psi, \xi)$ depends on $J, \psi$ but only on the sign of $\xi$. Firstiy $\mathrm{Eq}$. (28) shows that, to the $v^{1} \operatorname{order}, \frac{\partial h}{\partial \psi}(J, \psi, \xi)$ does not depend on $\xi$ except for its sign since $g_{0}$ has been shown to be independent of $\xi$. Secondly the fact that $h(U, \dot{\psi}, \xi)$ is even in u according to $E q$. (25) leads to the boundary condition at the turning point that $\lim _{u \rightarrow 0} \frac{\partial h}{\partial u}(u, \psi, \xi)=0$ or, using $J$ instead of $u$, that

$$
\lim _{\psi \rightarrow \psi} \sqrt{\mathrm{J}-\mathrm{Y}(\psi)} \frac{\partial h}{\partial J}(J, \psi, \xi)=0 .
$$

Thus both the differential equation and the boundary condition for $h$ are independent of $\xi$ if $h$ is self-consistently assumed to be so.

To find the flux at the spatial point $r$ we set $x=0$. Define

$$
\begin{gathered}
J_{2} \equiv x_{0}^{2} \xi^{2}+y(\psi)=J(x=0, \psi, \xi) . \\
h_{0}\left(J_{2}, \psi\right) \equiv[h(J, \psi, \xi)]_{x=0}, \quad g_{0}\left(J_{2}, \psi\right) \equiv\left[g_{0}(J, \psi, \xi)\right]_{x=0 .}
\end{gathered}
$$

It then follows from Eq. (28) that, to order $v^{\prime}$,

$$
\frac{\partial h_{\sigma}}{\partial \psi}\left(J_{2}, \psi\right)=-\frac{2 \sigma r x_{0}^{2} v_{i}}{\omega} \frac{\partial}{\partial J_{2}}\left[\sqrt{J_{2}-y(\psi)} \frac{\partial g_{0}}{\partial J_{2}}\left(J_{2}, \psi\right)\right] \text {. }
$$

Using $\mathcal{N}_{2}$ to replace $x_{0} \xi=\sigma \sqrt{J_{2-y^{y}}}$ as integration variable, so that

$$
\int_{-\infty}^{\infty} d \xi \longrightarrow-\int_{\infty}^{y(\psi)} \frac{d J_{2}}{2\left|x_{0}\right| \sqrt{J_{2}-y(\psi)}}+\int_{y(\psi)}^{\infty} \frac{d J_{2}}{2\left|x_{0}\right| \sqrt{J_{2}-y(\psi)}} .
$$


we obtain

$$
\begin{aligned}
& \frac{1}{2 \pi} \int_{-\pi}^{\pi} d \psi \int_{-\infty}^{\infty} d \xi \frac{\partial y}{\partial \psi} h\left(J_{2}, \psi\right)=\frac{1}{2 \pi\left|x_{0}\right|} \int_{-\pi}^{\pi} d \psi\left[\int_{\infty}^{y(\psi)} d J_{2} \frac{\partial \sqrt{J_{2}-y(\psi)_{h}}}{\partial \psi}\left(J_{2}, \psi\right)\right. \\
&\left.-\int_{y(\psi)}^{\infty} d J_{2} \frac{\partial \sqrt{\partial-y(\psi)}}{\partial \psi} h_{+}\left(J_{2}, \psi\right)\right]
\end{aligned}
$$

Integrating ty part and noting that the surface terms such as

$$
\int_{-\pi}^{\pi} d \psi \int_{y(\psi)}^{\infty} d J_{2} \frac{\partial}{\partial \psi}\left(\sqrt{J_{2}-y(\psi)} h_{+}\right)=\int_{-\pi}^{\pi} d \psi \frac{\partial}{\partial \psi} \int_{y(\psi)}^{\infty} d J_{2} \sqrt{J_{2}-y(\psi)} h_{+}=0
$$

where the first equality holds because $[h \sqrt{\sqrt{2-y}}]_{J_{2}=y}=0$ and the second holds because of periodicity, we obtain

$$
\frac{1}{2 \pi} \int_{-\pi}^{\pi} d \psi \int_{-\infty}^{\infty} d \xi \frac{\partial y}{\partial \psi} h\left(J_{2}, \psi\right)=\sum_{\sigma=+,-} \frac{1}{2^{\pi}\left|x_{0}\right|} \int_{-\pi}^{\pi} d \psi \int_{y(\psi)}^{\infty} d J_{2} \sqrt{J_{2}-y} \frac{\partial h_{\sigma}\left(J_{2}, \psi\right)}{\partial \psi}
$$

Upon substituting Eq. (40) into Eq. (42) and carrying out the subsequent integral over $\mathrm{J}_{2}$ Eq. (42) becomes

$$
\begin{aligned}
& \frac{1}{2 \pi} \int_{-\pi}^{\pi} d \psi \int_{-\infty}^{\infty} d \xi \frac{\partial y}{\partial \psi} h\left(J_{2}, \psi\right)=\sum_{\sigma} \frac{2 \sigma r\left|x_{0}\right| v_{1}}{\omega} \frac{1}{2 \pi} \int_{-\pi}^{\pi} d \psi \int_{y(\psi)}^{\infty} d J_{2} \sqrt{J_{2}-y} \frac{\partial}{\partial J_{2}}\left[\sqrt{J_{2}-y} \frac{\partial g_{0}}{\partial J_{2}}\right] \\
& =\sum_{\sigma} \frac{\sigma r\left|x_{0}\right|^{\nu} i}{\omega} \frac{1}{2 \pi} \int_{-\pi}^{\pi} d \psi g_{0}^{(t)}\left(s_{2}+\infty\right) \\
& =\sum_{0}^{o r\left|x_{0}\right| \nu_{i}} g_{0}^{(t)}\left(j_{2}+\infty\right)
\end{aligned}
$$


where we have used the facts that $g_{0}^{(b)}\left(J_{2}=y\right)=c$ and $g_{0}^{(t)}\left(J_{2} \rightarrow \infty\right)$ is independnet of $\psi$ according to Eq. (37). After some algebra and the use af Eq. $(32), g_{0}{ }^{(t)}\left(J_{2}+\infty\right)$ of Eq. (37) can be simplified as

$$
\lim _{j_{2} \rightarrow \infty} g_{0}^{(t)}=\sigma \frac{\pi}{2} \gamma\left|\frac{2 a_{1}}{\psi_{r}^{r}}\right|^{1 / 2} \frac{\partial f_{M}}{\partial r}
$$

where ${ }^{16}$

$$
\begin{aligned}
\gamma & =\int_{1}^{\infty} d u\left[\frac{1}{E_{-}(u)}-\frac{1}{\pi u}\right]-\frac{2}{\pi / 2} \\
& =\int_{0}^{\infty} d u\left[\frac{1}{E_{+}(u)}-\frac{1}{\pi u^{1 / 2}}\right],
\end{aligned}
$$

and

$$
E_{t}(u) \equiv \int_{-\pi / 2}^{\pi / 2} d \psi \sqrt{u \pm \sin ^{2} \psi} .
$$

Substitution of Eqs. (43), (44), (14) and $\omega=-r \psi_{r}^{-} / 2 t_{1}$ into Eq. (39) yields the averaged radial particle flux at distance $r$ from the mirror axis:

$$
\left\langle a_{r}\right\rangle=\frac{2 \sqrt{2} \pi^{2} \gamma H}{m_{i}^{2} L} \sum_{k=1,3,5, \ldots} \int_{d \mu t_{\| l}} \varepsilon_{k}^{2}(r, \mu) v_{i}(r, \mu) \frac{\left|a_{1}\right|^{1 / 2} \mid \psi_{\varepsilon}^{-}}{\left|\psi_{r}^{-}\right|^{3 / 2}} \frac{\partial f_{M}}{\partial r}
$$

Qualitatively the form of the diffusion coefficient can be arrived at by a random-walk type argument ${ }^{4}$ :

$$
0_{\text {res.ban }} \sim x_{\text {res }}^{2} \frac{\Delta \varepsilon}{\varepsilon} v_{\text {res }}^{\text {eff }} \sim\left|a_{1}\right|^{1 / 2} R^{3 / 2} v_{i}
$$

which ts seen to be consistent with Eq. (46). If we interpret $v_{i}(r, \mu)$ as the $90^{\circ}$-collision frequency, the form of Eq. (46) regarding the dependence on $v_{i}, a_{p}, \psi_{\varepsilon}, \psi_{r}^{-}$also agrees with the scaling ar sument of $R, H_{1}$ Cohen ${ }^{6}$ and oiffers in appearance from the result given in Ref. 3. However, when the 
detailed expression for $v_{i}(r, \mu)$ of Eq. (A7) is substituted into Eq. (46) the result can be converted after sone algebra into a rather different form

$$
\left\langle q_{r}\right\rangle-\sum_{k} \int_{d_{\mu} t_{\|}} \tilde{v}_{i}(r, \mu) \frac{\left|a_{1}\right|^{1 / 2}\left|\psi_{r}\right|^{1 / 2}}{\left|\psi_{E}\right|} \frac{\partial f_{\mu}}{\partial r} \text {. }
$$

This form agrees with that in Ref. 3. In fact, our converted $\tilde{v}(r, \mu)$ in Eq. (47) would be the same as $v_{i}^{R-S}(r, \mu)$ of Ryutov-Stupakov ${ }^{3}$ if the latter is corrected for some of its dependence on $\varepsilon_{k}$ and $\phi$. The lesson learned from the comparison of Eqs. (46) and (47) is that the form can vary from one formulation to another and, for quantitative purpose, the so-called collision frequency must be first calculated analytically.

For $\Delta \psi>1$ and in the regime of high collision frequency, $v_{i} t_{\|} \gg 1$, hardly any of the resonant particles and the MR-particles can complete the ir arbits. The diffusion is then dominated by the nonresonant ( $\Delta E \sim E$ ) paricles which constitute the majority. Each of these will have to random-walk from one transition region to the other before it receives another kick of order $a_{1}$. Since these kicks are phased randomly the diffusion coefficient is given by

$$
D_{\text {coll. }} \sim \frac{a_{1}^{2}}{\tau_{\text {ran.walk }}} \sim \frac{a_{1}^{2}}{t_{\|}^{2} v_{i}}
$$

where $\tau_{\text {ran walk }} \sim t_{i}{ }^{2} v_{i}$ is the time taken by a particle to random-walk from one end to the other. 
$\checkmark$ Remarks and Comments

The radial diffusion in tandem mirrors is similar to that in toroidal systems in many physical and mathenatical aspects. The role of the smallness parameter $\delta=\frac{\text { poloidal radius }}{\text { toroidal radius }}$ for the toroidal system is played by $\delta=a_{1} / R$ in the present case. The mathematical procedure of expanding into low-order terms of $\delta$ is carried out in both cases. As a first calculation, the major approximations made here are patterned after the pioneering toroidal work of Galeev and Sagdeev. ${ }^{5}$ For a more reined calculation, which may improve the numerical result sonewhat, especially for the plateau regime, the variational method of Rutherford, ${ }^{17}$ Rosenbluth and Hazeltine ${ }^{18}$ . and others ${ }^{19}$ should be followed

Finally we make a remark on the effect of the asymetry in the central solencidal section of the tandem system. The asymritetry arises from the nonaxial-symmetrical distribution of the particle density as well as of the resultant magnetic field as a result of particle diffusion between the transition region and the central region. This effect is also of the first order in the asymetry parameter and is indeed of importance in the neoclassical diffusion. ${ }^{2}$ Although it might be argued intuttively that this effect should be less important for resonant diffusion, a more careful analysis may be. worth doing. 


\section{ACKMOULEOGNENT}

I am grateful to L. D. Pearlstein for suggesting this investigation, to R. H. Cohen, H, Berk, D. Baldwin and S. Auerbach for helpful discussions, and especially to G. Smith for hłs patience and effort in answering many trivial questions, as well as in helping to educate a beginner in the field of magnetized plasma. 


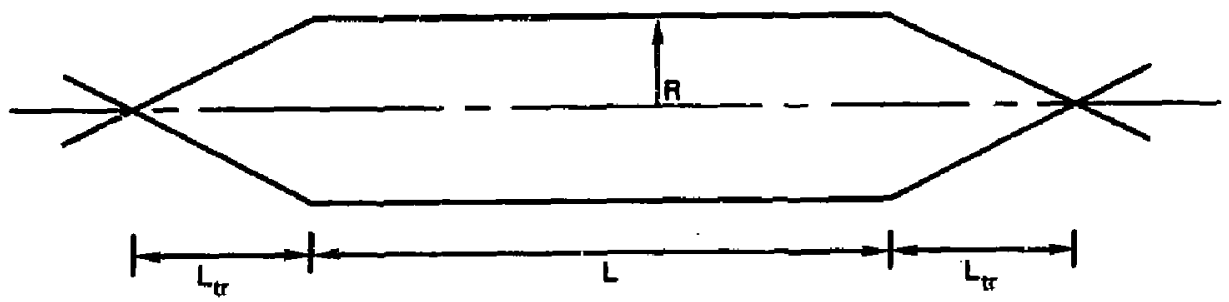

Fig. 1 The tandem mirror system - the central section and the transition regions.

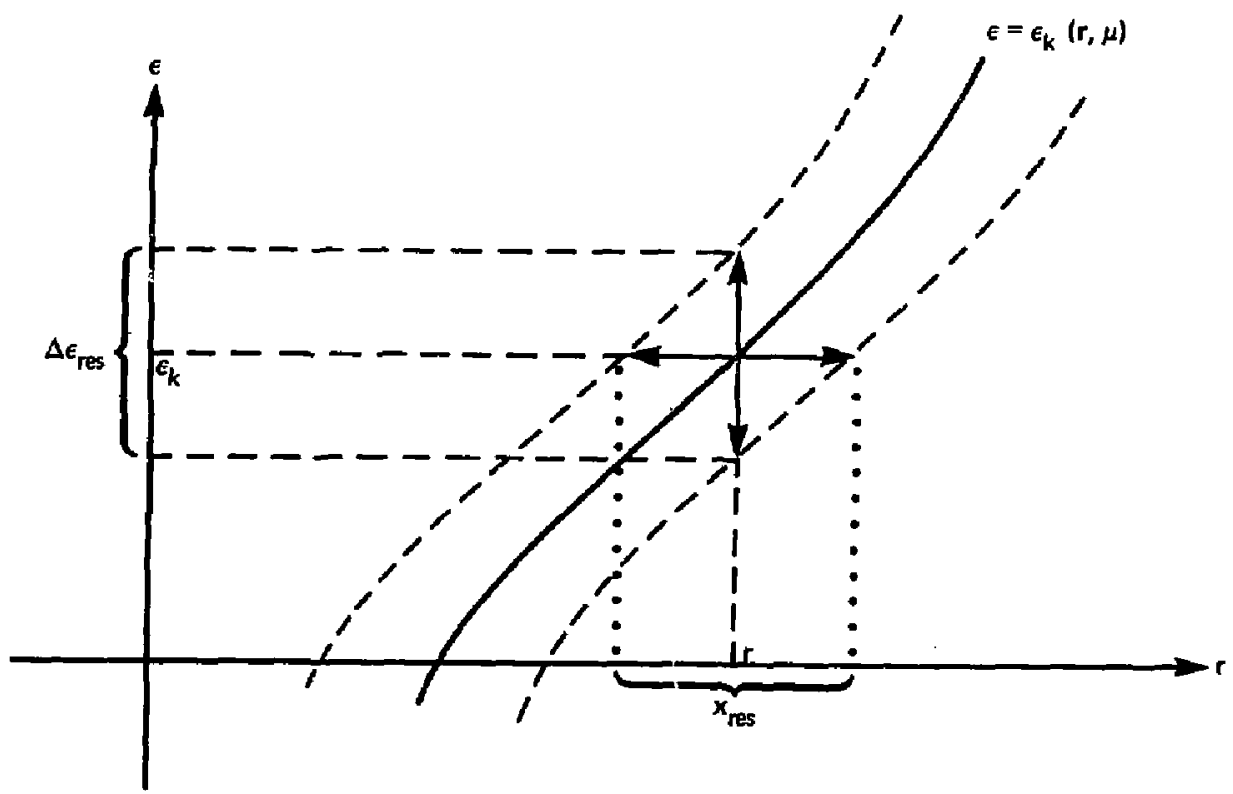

Fig. 2 The resonance region for a given $\mu$. 
APPENDIX

Derivation of the Collision Term in Eq. (24)

We start from the Landau form of collision integral

$$
\left(\frac{\partial f}{\partial t}\right)_{\text {col1. }}=-\frac{\partial}{\partial v_{\alpha}} \mathrm{J}_{\alpha}(\vec{v}) .
$$

where

$$
\begin{aligned}
J_{\alpha}(\vec{v}) & =A \int d \vec{v},\left(\frac{\delta \beta}{u}-\frac{u_{\alpha} u_{B}}{u^{3}}\right)\left[\frac{\partial f(\vec{v})}{\partial v_{\beta}} f(\vec{v})-f\left(\vec{v}^{-}\right) \frac{\partial f\left(v^{-}\right)}{\partial v_{B}}\right], \\
\vec{u} & =\vec{v}-\vec{v}, \\
A & =-\frac{n_{0} e^{4}}{m_{i}^{2}} \log \left[\frac{T_{i}^{3}}{4 \pi n_{0} e^{6}}\right] .
\end{aligned}
$$

Approximating ${ }^{5}$ the background particles $f\left(v^{-}\right)$with which the $\vec{v}$-particle being considered is colliding by $f_{M}\left(v^{-}\right)$we then replace $f(\vec{v})$ by $\delta f(\vec{v})$ to obtain 20

$$
\begin{aligned}
J_{\alpha}(\vec{v}) & =-A \int \alpha \vec{v} \cdot\left(\frac{\delta \alpha \beta}{v}-\frac{u_{\alpha} u_{\beta}}{u^{3}}\right)\left[\frac{\delta f(\vec{v}) v_{\beta} f_{M}(\vec{v} \cdot)}{T_{i}}+\frac{f_{M}(\vec{v}-)}{m_{i}} \frac{\partial \delta f(\vec{v})}{\partial v_{\beta}}\right] \\
& =-A I_{\alpha \beta}(\vec{v})\left[v_{\beta} \frac{\delta f(\vec{v})}{T_{i}}+\frac{1}{m_{j}} \frac{\partial \delta f(\vec{v})}{\partial v_{B}}\right]
\end{aligned}
$$

where

$$
\begin{aligned}
I_{\alpha \beta}(\vec{v}) & \equiv \int d \vec{v}-\frac{u^{2} \delta_{\alpha \beta}-u_{\alpha} u_{\beta}}{u^{3}} f_{M}(\vec{v}-) \\
& =\frac{\partial^{2}}{\partial v_{\alpha} \partial v_{\beta}} \int d \vec{v} \cdot u f_{M}\left(v^{-}\right)
\end{aligned}
$$

Using the explicit form of the local Maxwellian distribution function

$$
f_{M}\left(v^{\prime}\right)=G e^{-g v^{-2}}
$$


24

and after some algebra can reduce $I_{\alpha \beta}\left(\vec{v}^{*}\right)$ to

$$
\begin{gathered}
I_{\alpha \beta}(\vec{v})=\frac{2 \pi G}{g}\left\{\left(\frac{\delta_{\alpha \beta}}{v}-\frac{v_{\alpha} v_{\beta}}{v^{3}}\right)\left[-\frac{1}{v^{2}} \int_{0}^{v} d y y^{2} e^{-g y^{2}}+\int_{0}^{v} d y e^{-g y^{2}}\right]\right. \\
\left.+\frac{v_{\alpha \beta} v_{\beta}}{v^{3}} \frac{2}{v^{2}} \int_{0}^{v} d y y^{2} e^{-g y^{2}}\right\}
\end{gathered}
$$

(AM)

Substituting Eqs. (AA), (A2) into (Al) and after some simplication we find 5

$$
\begin{gathered}
\left(\frac{\partial f}{\partial t}\right)_{\operatorname{col1} .}=\frac{2 \pi \lambda e^{4}}{m_{i}^{2}} \frac{\partial}{\partial v_{\alpha}}\left\{\left[\left(\eta(x)+\eta^{-(x)}-\frac{\eta(x)}{2 x}\right)\left(\frac{\alpha \beta}{v}-\frac{v_{\alpha} v_{\beta}}{v^{3}}\right)+\frac{v_{\alpha} v_{B}}{v^{3}} \cdot \frac{\eta(x)}{x}\right],\right. \\
\cdot\left(\frac{\partial \delta f(v)}{\partial v_{B}}+\frac{2 v_{\beta}}{\left.\left.v_{T}^{2} \delta f(t)\right)\right\}}\right.
\end{gathered}
$$

where

$$
\begin{aligned}
x & \equiv\left(\frac{v}{v_{T}}\right)^{2}=\frac{\varepsilon-e \phi}{T_{i}}, n(x) \equiv \frac{2}{\sqrt{\tau_{i}^{-}}} \int_{0}^{x} d t t^{1 / 2} e^{-t}, \\
n^{-}(x) & =\frac{d n}{d x}=\frac{2}{\sqrt{\pi}} x^{1 / 2} e^{-x}, \quad \frac{m_{i} v_{T}^{?}}{2} \equiv T_{i}, \\
2 \lambda & =n_{0} \log \left[\frac{T_{i}^{3}}{4 \pi_{0} e^{6}}\right] .
\end{aligned}
$$

We shall now change variables from $\left(v_{x}, v_{y}, v_{z}\right)$ to $(\xi, \mu, \theta)$ where

$$
\begin{aligned}
& v_{x}=\sqrt{2 j s / m_{i}} \cos \theta, \quad v_{y}=\sqrt{2 \mu H / m_{i}} \sin \theta, \mu H=\frac{1}{2} m_{i} v_{1}^{2}, \\
& v_{z}=\sqrt{\left(2 / m_{i}\right)(\varepsilon-e \phi-\mu H)}, \quad \xi=\left(\varepsilon-\varepsilon_{k}(r, \mu)\right) / \varepsilon_{k}
\end{aligned}
$$

and keep only the $\frac{\partial^{2} \delta f}{\partial \xi^{2}}$ terms in (A5). Thus, 
25

$$
\begin{aligned}
\left(\frac{\partial f}{\partial t}\right)_{c o 11 .} & \approx \frac{2 \pi \lambda e^{4}}{m_{i}^{2}}\left\{\left(\eta+\eta^{\prime}-\frac{\eta}{2 x}\right) \frac{1}{v^{3}}\left(v^{2} \delta_{\alpha \beta}-v_{\alpha} v_{\beta}\right)+\frac{1}{v^{3}} v_{\alpha} v_{\beta} \frac{n}{x}\right\} \frac{\partial^{2} \delta f}{\partial v_{\alpha} \partial v_{\beta}} \\
& \approx \frac{2 \pi \lambda e^{4}}{v^{3} \varepsilon_{k}^{2}} \frac{\partial^{2} \delta f}{\partial \xi^{2}}\left\{\left(\eta+\eta^{-}-\frac{\eta}{2 x}\right)(1-a)^{2} v_{\perp}{ }^{2} v_{z}^{2}+\frac{\eta}{x}\left(a v_{\perp}{ }^{2}+v_{z}^{2}\right)^{2}\right\}
\end{aligned}
$$

where $a \equiv 1-\frac{1}{H} \frac{\partial \varepsilon_{k}}{\partial \mu}(r, \mu)$. We may also rewrite Eq. (A6) in the form of Eq. (24),

$$
\left(\frac{\partial f}{\partial t}\right)_{\text {coll. }}=v_{i}(r, \mu) \frac{\partial^{2} \delta f}{\partial \xi^{2}},
$$

where

$$
\begin{aligned}
& v_{i}(r, \mu)=4 \sqrt{\frac{2 \pi}{m_{i}}} \frac{\lambda e^{4}\left(\varepsilon_{k}-e \phi\right)^{3 / 2} \cdot \mu^{2} H^{2}}{\varepsilon_{k}^{2} T_{i}^{3}} . \\
& \cdot\left[\left(\frac{\varepsilon_{k}-e \phi-\mu H}{\mu H}\right)(1-a)^{2} G\left(\frac{\varepsilon_{k}-e \phi}{T_{i}}\right)+\left(a+\frac{\varepsilon_{k}-e \phi-\mu H}{\mu H}\right)^{2} F\left(\frac{\varepsilon_{k}-e \phi}{T_{i}}\right)\right], \\
& G(x)=\frac{1}{x^{3}}\left[\left(1-\frac{1}{2 x}\right) \int_{0}^{x} e^{-t} t^{1 / 2} d t+e^{-x} x^{1 / 2}\right], \\
& F(x)=\frac{1}{x^{4}} \int_{U}^{x} e^{-t} t^{1 / 2} d t .
\end{aligned}
$$




\section{REFERENCES}

1. D. D. Ryutoy and G. V. Stupakov; Pis ma Zh. Eksp. Teor 26, 186 (1977); trans. in Sov. Phys. JE.P Lett 26, 177 (1977).

2. D. D. Ryutov and G. Y. Stupakov; Fix. Plasmy 4, 501 (1978), Lawrence Livermore Laboratory trans. 02544.

3. D. 0. Ryutov and G. V. Stupakov, Dok1, Akad. Nauk, 240, (1978); Lawrence Livermore Laboratory trans. 0¿548.

4. D. E. Baldwin, Lawrence Livermore Laboratory UCID-17926, Sept. 15, 1978

5. A. A. Galeev and R. Z. Sagdeev, Zh. Eksp. Teor. Fiz. 53, 348 (1967) Sov. Phys. - JETP 26. 233 (1968).

6. R. Cohen (private comunication).

7. This result justifies the neglect of $\psi_{r}^{+} x \ll \psi_{\varepsilon}^{-} \Delta \varepsilon_{\text {, }}$ making the derivation of Eq. (17) self-consistent.

8. E. A. Frieman, Phys. Fluids $\underline{13}, 490$ (1970).

9. T. E. Stringer, Phys. Fluids 13, 810 (1970).

10. S. P. Auerbach, Phys. Fluids 20, 1836 (1977).

11. He can actually perform the transformation from $(x, \psi, \xi)$ to $(J, \psi, \xi)$ directly. The purpose of introducing the variable in the intermediate step is to establish Eq. (26). 
12. Our Eqs. (27) and (28) differ from the corresponding ones in the treatment of the neocTassical diffusion, given as Eqs. (62) and (63) in Ref. 2, although our Eq. (24) has the same form as their Eq. (58). If we define $J_{2} \equiv x_{0}^{2} \xi^{2}+\dot{y}(\psi)=J(x=0, \psi, \xi)$, then at $x=0$ we have $\frac{\partial h\left(\psi, J_{2}\right)}{\partial \psi}=\left[\frac{\partial h}{\partial \psi}(J, \psi, \xi)+\frac{\partial h}{\partial \xi}\left(J_{2},, \xi\right) \frac{\partial \xi\left(J_{2}, \psi\right)}{\partial \psi}\right]_{x=0} \neq\left[\frac{\partial h\left(J_{,}, \psi_{2} \xi\right)}{\partial \psi}\right]_{x=0}$ or $J=J_{2}, x_{0} \xi=\sigma \sqrt{J_{2}-y}$

Apparent ly the $\left(\frac{\partial h}{\partial \xi} \frac{\partial \xi}{\partial \psi}\right)_{x=0}$ term seems to be missing from their Eq. (62).

13. Again we note that the corresponding equations in Ref. 2, their Eqs. (62) and (63), involve only two variables, $j_{2} \equiv x_{0}^{2} \xi^{2}+y(\psi)$ and $\psi$. This reduction of three variables $x, \psi, \xi$ to two variables $J_{2}, \psi$ was apparently arrived at by setting $x=0$ in their Eq. (61) which involves $\frac{\partial h}{\partial x}$ and $\frac{\partial g}{\partial x}$.

14. B. N. Breizman, V. V. Mirnov, and D. D. Ryutov, Zh. Eksp. Tear. Fix. 58, 1770 (1970) Sav. Phys. - JETP 31. 948 (1970) .

15. In terms of the variables $x, \psi, \xi$, we note that $g_{0}(x, \psi, \xi)$ evaluated at $x=0$ or $\sqrt{\mathrm{j}-y}=\left|x_{0} \xi\right|$ does depend on $\xi$ according to Eqs. (32) and $(36)$.

16. The demonstration of the equality of the two integral expressions for $y$ as we $h 1$ as the numerical value $\gamma=-0.44$ are due to $R$. H. Cohen.

17. P. H. Rutherford, Phys. Fluids 13, 482 (1970) 
18. M. N. Rosenbluth and R. D. Hazeltine, Phys. Fluids $\underline{15}, 116(i: / 2\}$.

19. For a review of torofdal transport, see F, L. Hinton and R. D. Hazeltine, Rev. Mod. Phys. 48, 239 (1976).

20. The approximation (A2) to the collision integrai has been improved upon by Kovnzhnykh in JETP 29, 475 (1969) and by P. H. Rutherford in Ref. 17. 